\title{
Gene Expression Profiles for Recurrence of Lymph Node-positive Primary Breast Cancer in Women Over 40 Years of Age
}

\section{Sean Si Qian Ma}

Fudan University

Luyi Ye

Fudan University

Fan Zhang

Shanghai OE Biotech Co., Ltd

Tiansheng $\mathrm{Xu}$

Shanghai OE Biotech Co., Ltd

\section{Zai-Si Ji}

Fudan University

Enuo Liu ( $\nabla$ liuenuo@sippr.org.cn )

Shanghai Ocean University https://orcid.org/0000-0001-6146-0797

Research article

Keywords: breast cancer, lymph node metastasis, olfactory receptor

Posted Date: September 17th, 2021

DOl: https://doi.org/10.21203/rs.3.rs-880067/v1

License: (9) (i) This work is licensed under a Creative Commons Attribution 4.0 International License. Read Full License 


\section{Abstract}

\section{Background:}

Specific gene expression profiles correlate with recurrence of breast cancer in lymph node-negative patients. In contrast, insufficient knowledge is available regarding tumor-specific gene expression in patients with lymph node metastasis before surgery. Furthermore, such patients experience cumulative incidences of relapse greater than $50 \%$.

\section{Methods:}

Sections of formalin-fixed paraffin embedded (FFPE) were prepared from breast tumors of 37 patients who were followed for at least 5 years. FFPE samples of patients with recurrent ductal breast cancer $(\mathrm{n}=$ 25) and 12 FFPE samples of such patients without recurrence were subjected to microarray analysis to identify gene expression profiles specifically associated with positive lymph nodes confirmed during surgery that were accompanied by lymphocytic invasion. Immunohistochemistry was employed to determine the estrogen receptor (ER) status of cancer tissues. All patients were administered tamoxifen after surgery, and this treatment continued for more than 5 years, or until cancer recurred. This strategy eliminated interactions between different therapeutics as potential confounding factors that influenced patients' outcomes.

\section{Results:}

Sixteen genes were expressed at significantly higher levels in patients with ER-positive (+) breast cancer with recurrence compared with those without recurrence. Gene Set Enrichment Analysis of The Kyoto Encyclopedia of Genes and Genomes (KEGG) identified 73 genes encoding olfactory receptors included in the "Olfactory transduction" pathway that were enriched in the ER+ recurrence group (FDR $P<0.05$ ). The KEGG "Histidine metabolism" and "Retinol metabolism" pathways were enriched in patients with ERnegative $(-)$ breast cancer with recurrence (FDR $P<0.05)$.

\section{Conclusions:}

The present study is the first, to our knowledge, to identify 16 genes encoding proteins with diverse functions as well as 73 genes encoding olfactory receptors. These genes may serve as presurgical biomarkers for the recurrence of ER+ breast cancers with lymph node metastasis before surgery. These findings identify potential therapeutic targets for preventing cancer relapse, particularly after lymph nodes metastasis.

\section{Background}

Advances in the treatment of breast cancer have decreased the rate of isolated locoregional recurrence [1]. For example, lumpectomy followed by radiotherapy reduces the recurrence of ipsilateral breast tumors by $52 \%$ compared with lumpectomy alone, and lumpectomy followed by radiotherapy and tamoxifen 
treatment reduces the incidence of recurrence by $32 \%$ compared with lumpectomy followed by radiotherapy [2]. Furthermore, recent studies show that the overall response rates of patients with breast cancer treated with immune checkpoint inhibitors are similar to those of numerous solid malignancies, which may identify effective immunotherapy-based combination strategies [3]. Moreover, young age, node positivity, larger tumors, and hormone receptor-negative tumors are associated with a higher risk of recurrence $[4,5]$. Tumor biology influences the time interval to isolated locoregional recurrences. For example, the median times to isolated locoregional recurrences of breast cancer, measured over 20 years [6], are as follows: ER-positive (+), 6.8 years; ER-negative (-), 3.6 years [7].

Reverse-transcriptase-polymerase-chain-reaction assays of 21 prospectively selected genes expressed by $\mathrm{ER}+$ patients revealed a correlation between the likelihood of distant recurrence with lymph node-negative breast cancer treated with tamoxifen [8]. Furthermore, the 21-Gene Recurrence Score serves as an independent prognosticator for time to first progression and 2-year overall survival experienced by patients aged 21-70 years with de novo stage IV ER + breast cancer [9] as well as for women $<40$ years of age with node-negative and limited node-positive breast cancer [10].

A 76-gene signature comprising 60 genes expressed by ER + patients and 16 genes expressed by ERpatients identifies patients at high risk of distant recurrence [11]. Although the overall prognosis of ERbreast cancer is worse compared with that of the ER + subtype, not all patients with ER- breast cancer experience poor clinical outcomes. These prognostic signatures are very useful for determining the risk of recurrence in the ER + subgroup, although they are less informative for ER- and erb-b2 receptor tyrosine kinase $2+$ breast cancers [12]. Other microarray studies show the differences in recurrence frequencies associated with gene expression between $\mathrm{ER}+$ and ER- patients with breast cancer. The immune response and tumor invasion are associated with patients with ER-/HER2- and HER2 + breast cancer, respectively [13]. The heterogeneity in the clinical outcomes of ER- breast cancer involves differences in the expression levels of genes that encode the components of the complement and immune response pathways, independent of lymphocytic infiltration [14]. Furthermore, among these pathways, the immune response is the most prognostic, as indicated by the expression levels of genes that encode complement C1q A chain (C1QA); immunoglobulin lambda constant 2 (IGLC2), LY9 (lymphocyte antigen 9); TNF receptor superfamily member 17 (TNFRSF17); secreted phosphoprotein 1 (SPP1); X-C motif chemokine ligand 2 (XCL2); and human leukocyte antigen F (HLA-F) [14].

Although these predictors perform well in identifying relapse of patients without involved lymph nodes, they fail to predict patients with positive lymph nodes. Furthermore, these reports analyzed the populations of Europe or the United States. In contrast, limited data are available for populations of Asia. For example, a study conducted in China found that Ki-67 expression levels are significantly associated with early relapse of breast cancer [15].

Here we compared the gene expression profiles of tumor tissue of patients with ductal breast cancer, with or without recurrence, within 5 years after surgery. Both groups of patients had positive lymph nodes confirmed during surgery, which were accompanied by lymphocytic invasion. Thus, we aimed here to 
identify gene expression patterns that will contribute to improving therapy designed to prevent the recurrence of breast cancer in patients with lymphocytic invasion who are administered chemotherapy and tamoxifen.

\section{Methods}

\section{Study design and subjects}

The present study included 37 patients who underwent breast cancer surgery at Huangpu District Central Hospital of Shanghai, China from 2005 to 2008. These patients were followed for more than 5 years. Sections of formalin-fixed paraffin-embedded (FFPE) tissues were prepared from breast tumors. The FFPE tissue samples included those of 25 of patients with ductal breast cancer with recurrence (ER+/ER-; $n=16, n=9$, respectively), and 12 of patients with ductal breast cancer without recurrence $(E R+/ E R-; n=6, n=6$, respectively). ER expression was detected using immunohistochemistry employing a monoclonal antibody against ER. Histologic grade was assessed according to the World Health Organization criteria. All patients had positive lymph nodes confirmed during surgery, which were accompanied by lymphocytic invasion. These patients were administered tamoxifen starting immediately after surgery and continuing for more than 5 years or until cancer recurrence. All patients received chemotherapy, thereby eliminating potential interactions between different treatments to exclude the influence of potential confounding factors on outcomes.

\section{RNA extraction, microarray hybridization, and data analysis}

A RecoverAll Total Nucleic Acid Isolation Kit AM1975 (Thermo Scientific, USA) was used to extract total RNA from FFPE tissues according to the manufacturer's protocol. The process mainly involved deparaffinization, protease digestion, nucleic acid isolation, DNase digestion, and final purification. RNA purity and quantification were evaluated using a NanoDrop 2000 Spectrophotometer (Thermo Scientific, USA). RNA integrity was assessed using an Agilent 2100 Bioanalyzer (Agilent Technologies, Santa Clara, CA, USA).

Gene expression profiles were determined using an Affymetrix Human Clariom D Assay (OE Biotechnology Co., Ltd., Shanghai, China). Sample labeling, microarray hybridization and washing were performed according to the manufacturer's standard protocols. Briefly, total RNAs were reversetranscribed and purified. Next, the second-cycle cDNAs were fragmented and labeled with biotin, and the labeled cDNA samples were used to probe the microarrays. After washing and staining, the microarrays were scanned using an Affymetrix Scanner 3000 (Affymetrix).

Affymetrix GeneChip Command Console software (version 4.0, Affymetrix) was used to extract raw microarray data, and Expression Console software (version 1.3.1, Affymetrix) was used to perform Robust Multi-array Average (RMA) normalization for gene expression analysis. Differentially expressed genes were identified using GeneSpring software (version 13.1, Agilent Technologies). The fold-change in expression level was used to compare groups of microarray samples. Heat maps generated using the 
clustermap function of the Seaborn package in Python visualized gene expression levels among samples. The microarray data were deposited in the Gene Expression Omnibus (GEO) database (accession code GSE183231).

\section{Gene set enrichment analysis (GSEA)}

GSEA [16] ranks all genes in a dataset according to their differential expression. To test the significance of a gene set, an enrichment score is defined as the maximum distance from the middle of the ranked list. Thus, the enrichment score indicates whether the genes contained in a gene set are clustered toward the beginning or end of the ranked list. Self-contained and competitive hypothesis tests are conducted using GSEA by altering randomization for hypothesis testing. To develop a self-contained hypothesis, the phenotype labels are permuted; in contrast, the genes are permuted for a competitive hypothesis. We performed 1000 permutations to estimate empirical $P$ values of the gene sets. The data were acquired from the GSEA Molecular Signatures Database v7.4 (http://www.gsea$\mathrm{msigdb}$.org/gsea/msigdb/index.jsp). The threshold for identifying significant pathways and entries was defined by the false discovery rate FDR $(P<0.05)$ and the normalized enrichment score $|\mathrm{NES}|>1$.

\section{Results}

\section{Clinical and pathological features of breast cancer patients}

Among 37 patients included in the present study, 11 showed evidence of distant metastasis, 14 showed local recurrence, 12 died after a previous relapse, and 3 patients died without evidence of disease. The 16 ER + patients who relapsed and the 9 ER-patients were followed for 1482 days and 1115 days, respectively. Clinical and pathological features of the patients are summarized in Table 1. The ER + and ER- recurrence groups (local and distant) had slightly higher proportions of larger tumors compared with those of the nonrecurrence ER + and ER- control groups, respectively. There were no significant differences among the groups in age and number of positive lymph nodes observed during surgery. 
Table 1

Clinical and pathological features of 37 patients in surgery

\begin{tabular}{|c|c|c|c|c|}
\hline & $\begin{array}{l}\text { ER + recurrence } \\
\text { recurrence (local } \\
\text { and distant) }\end{array}$ & $\begin{array}{l}\text { ER+ } \\
\text { nonrecurrence } \\
\text { control }\end{array}$ & $\begin{array}{l}\text { ER-recurrence } \\
\text { recurrence } \\
\text { (local and } \\
\text { distant) }\end{array}$ & $\begin{array}{l}\text { ER- } \\
\text { nonrecurrence } \\
\text { control }\end{array}$ \\
\hline Subjects (n) & 16 & 6 & 9 & 6 \\
\hline Age in surgery (year) & $56 \pm 13$ & $53 \pm 6$ & $56 \pm 12$ & $56 \pm 11$ \\
\hline $\begin{array}{l}\text { Time to first recurrence } \\
\text { (days) }\end{array}$ & $1482 \pm 650$ & - & $1115 \pm 754$ & - \\
\hline $\begin{array}{l}\text { lymphocytic invasion } \\
+/++(n)\end{array}$ & $10 / 6$ & $5 / 1$ & $3 / 6$ & $4 / 2$ \\
\hline positive nodes & $5.8 \pm 7.1$ & $5.2 \pm 2.7$ & $4.8 \pm 9.6$ & $4.0 \pm 5.0$ \\
\hline $\begin{array}{l}\text { Chemotherapy post } \\
\text { surgery }\end{array}$ & $\sqrt{ }$ & $\sqrt{ }$ & $\sqrt{ }$ & $\sqrt{ }$ \\
\hline Dead (n) & 8 & 2 & 4 & 2 \\
\hline $\begin{array}{l}\text { Premenopausal/ } \\
\text { Postmenopausal/ Lost (n) }\end{array}$ & $3 / 9 / 4$ & $0 / 4 / 2$ & $2 / 4 / 3$ & $2 / 0 / 4$ \\
\hline Tumor size $>20 \mathrm{~mm}(\mathrm{n})$ & 9 & 1 & 5 & 2 \\
\hline tumor size $\leqq 20 \mathrm{~mm}(\mathrm{n})$ & 14 & 5 & 4 & 2 \\
\hline Tumor size $(\mathrm{mm})$ & $29.3 \pm 15.1$ & $24.5 \pm 5.9$ & $31.0 \pm 15.3$ & $21.2 \pm 10.2$ \\
\hline Tamoxifen & used & used & used & used \\
\hline grade 3 / (grade 2and 1) & 0.30 & 0.00 & 0.55 & 1.00 \\
\hline
\end{tabular}

\section{Gene expression profiling of breast cancer tissues}

Gene expression profiles were determined using RNA samples from breast tumor tissues of patients as follows: $16 \mathrm{ER}$ + and $6 \mathrm{ER}$ + with or without recurrence, respectively, $9 \mathrm{ER}-$ with recurrence, and $6 \mathrm{ER}-$ without recurrence. The Affymetrix Clariom D Human Assay containing 135,750 probe sets, including 18,858 Entrez Gene RNAs and 66,845 IncRNAs. Principal component analysis (PCoA) revealed that the difference between the expression profiles of ER- with or without recurrence was greater than that between the corresponding two groups of ER + samples (Fig. 1).

Differences in gene expression profiles between ER + tumor tissues of patients with and without recurrence with positive lymph nodes 
When we arbitrarily defined a differentially expressed gene (DEG) according to a $>1.5$-fold change in expression compared with the reference $(P<0.05)$, we identified 1962 DEGs between the ER + with recurrence group compared with the ER + without recurrence group. Among 771 upregulated DEGs, including 116 coding genes, 392 noncoding genes, and 263 other genes, aspartate beta-hydroxylase (ASPH) was expressed at significantly higher levels (2.75-fold) in the ER + recurrence group compared with the ER + nonrecurrence group $(P<0.05)$. Phospholipase A2, group V (PLA2G2) was expressed at a significantly higher level (2.06-fold in the ER + recurrence group compared with the ER + nonrecurrence group, $P<0.05)$. Among 1191 downregulated $D E G s$ expressed by the $E R+$ recurrence and $E R+$ nonrecurrence groups, we identified the DEGs as follows: 203 coding, 555 noncoding, and 433 others (Fig. 2A, B). Part of the upregulated coding DEGs are shown in Table 2.

Table 2

Sixteen significantly different-expressed coding genes between ER positive samples with recurrence vs ER positive samples without recurrence (up-regulated fold change $>1.5$, p value $<0.05$ )

\begin{tabular}{|c|c|c|c|c|}
\hline $\begin{array}{l}\text { Fold } \\
\text { Change }\end{array}$ & P-val & $\begin{array}{l}\text { FDR P- } \\
\text { val }\end{array}$ & $\begin{array}{l}\text { Gene } \\
\text { Symbol }\end{array}$ & Description \\
\hline 2.75 & 0.0005 & 0.4937 & ASPH & Aspartate beta-hydroxylase \\
\hline 2.06 & 0.0000 & 0.2176 & PDCD6IP & programmed cell death 6 interacting protein \\
\hline 2.06 & 0.0049 & 0.6367 & PLA2G5 & phospholipase A2, group V \\
\hline 2.04 & 0.0028 & 0.6035 & PDIA6 & protein disulfide isomerase family A, member 6 \\
\hline 2.03 & 0.0409 & 0.7896 & ATP5I & $\begin{array}{l}\text { ATP synthase, } \mathrm{H}+\text { transporting, mitochondrial Fo } \\
\text { complex subunit } \mathrm{E}\end{array}$ \\
\hline 1.95 & 0.0001 & 0.3837 & DCP1B & decapping mRNA 1B \\
\hline 1.92 & 0.0138 & 0.7081 & MTF2 & $\begin{array}{l}\text { metal response element binding transcription factor } \\
2\end{array}$ \\
\hline 1.70 & 0.0028 & 0.6035 & GLS & glutaminase \\
\hline 1.69 & 0.0031 & 0.6135 & OR7E101P & $\begin{array}{l}\text { olfactory receptor, family } 7 \text {, subfamily E, member } 101 \\
\text { pseudogene }\end{array}$ \\
\hline 1.61 & 0.0039 & 0.6215 & ALG10 & ALG10, alpha-1,2-glucosyltransferase \\
\hline 1.60 & 0.0038 & 0.6182 & KLKB1 & kallikrein B1 \\
\hline 1.59 & 0.0027 & 0.602 & TEKT3 & tektin 3 \\
\hline 1.57 & 0.0031 & 0.6135 & $\mathrm{DHX} 8$ & DEAH (Asp-Glu-Ala-His) box polypeptide 8 \\
\hline 1.53 & 0.0150 & 0.716 & PIEZO2 & piezo-type mechanosensitive ion channel component \\
\hline 1.51 & 0.0039 & 0.6215 & CNTN1 & contactin 1 \\
\hline 1.50 & 0.0109 & 0.6962 & MCUR1 & mitochondrial calcium uniporter regulator 1 \\
\hline
\end{tabular}


GO enrichment analysis revealed that the DEGs were associated with the GO terms as follows: "Biological Process" ( $n=57)$, "Cellular Component" $(n=17)$, and "Molecular Function" $(n=22)$. The top $20 \mathrm{GO}$ terms are shown in Fig. 3A. The enriched pathways included 14 KEGG pathways associated with all DEGs (Fig. 3B). GSEA identified enriched KEGG pathways significantly associated with the recurrence group as follows: "Olfactory transduction" (FDR, $P=0.035$; NES $=1.45$ ), as well as the enriched GO term "DNA binding transcription factor activity" (FDR, $P=0.014$, NES $=-2.03$ ) that was associated with the nonrecurrence group (Fig. 3C, D).

\section{Differences in gene expression profiles between lymph node-positive ER- tumor tissue of patients with or without recurrence}

We identified 3833 DEGs between the ER- groups with or without recurrence, among which 1761 were upregulated and 2072 were downregulated (Fig. 4A). The DEGs included 208 coding genes, 934 noncoding genes, and 619 other genes. The latter DEGs included 343 coding genes, 815 noncoding genes, and 914 other genes (Fig. 4B).

GO enrichment analysis revealed that the DEGs were associated with $89 \mathrm{GO}$ terms, including 51 in "Biological Process", 20 in "Cellular Component." and 18 in "Molecular Function". Furthermore, there were 36 KEGG pathways associated with the DEGs between the ER- recurrence and nonrecurrence groups (Supplemental file S1).

GSEA identified the significantly enriched KEGG pathways "Histidine metabolism" and "Retinol metabolism" associated with the recurrence group (FDR $P<0.05$, NES $>1.0$ ) (Fig. 5A, B), and eight enriched GO terms were significantly associated with the nonrecurrence group (FDR $P<0.05$, NES $<-2.0$ ) (Supplemental file 2).

\section{Discussion}

Few reports contribute insights regarding predictors of outcomes or treatment targets applicable to women aged over 40 years with lymph node-positive primary breast cancer studied here. The $55 \%$ probability of recurrence associated with positive lymph nodes after surgery emphasizes the importance of developing more effective biomarkers [17]. Here we show, for the first time to our knowledge, that 16 genes encoding proteins with diverse functions and 73 genes encoding olfactory receptors may serve as biomarkers of the recurrence of breast cancer with the ER + phenotype accompanied by lymph nodes metastasis before surgery.

Diverse human tissues express olfactory receptors that contribute to physiological processes such as cell migration, proliferation and secretion and serve as biomarkers for carcinomas of the prostate, lung, small intestine and breast [18]. Olfactory receptor family 51 subfamily E member 2 (OR51E2) serves as a biomarker for prostate cancer. OR51E2 is also called prostate-specific G-protein-coupled receptor (PSGR), 
because it is upregulated in prostate cancer $[19,20]$. OR51E1, which is a paralog of OR51E2, serves as a potential biomarker for small intestine neuroendocrine carcinomas [21] and certain types of lung cancer [22]. Moreover, 111 olfactory receptors are detectable in testis, including specific expression of olfactory receptor family 4 subfamily $\mathrm{N}$ member 4 (OR4N4), and several other olfactory receptors are expressed in Epstein-Barr virus-transfected lymphocytes [23]. The present study is the first, to our knowledge, to report a correlation between the expression of olfactory receptor genes (73 gene set) and tumor recurrence of $E R+$ breast patients with breast cancer with lymph nodes metastasis. This discovery suggests that olfactory receptors may serve as predictors of recurrence of ER + breast cancer, particularly subsequent to lymph node metastasis.

ASPH is highly expressed during fetal development and in placental trophoblasts, but not in healthy adult human tissues. Furthermore, ASPH is specifically upregulated in cancer cells and is overexpressed in more than 20 different solid neoplasms, in which it contributes to the malignant phenotype. Moreover, ASPH is associated with increased cell proliferation, invasiveness, and poor prognosis. Metastasis is a major cause of death of patients with cancer, and new antimetastatic strategies are required. Numerous pathways contribute to cell migration and invasion of normal tissues by malignant tumors. ASPH is a key player in the malignant transformation of solid tumors through enhancing cell proliferation, migration, and invasion. Furthermore, ASPH promotes tumor growth through stimulation of angiogenesis and immunosuppression. These effects are mainly achieved via the activation of the Notch and SRC signaling pathways. ASPH expression is upregulated by growth factors and hypoxia in different human tumors, and its inactivation may have broad clinical effects. Moreover, ASPH expression is silenced in normal adult breast tissue, upregulated in malignancies in situ, and highly expressed in invasive ductal carcinoma [24].

Here we show that higher differential expression of ASPH correlated with tumor recurrence in ER + patients with breast cancer, including those treated with tamoxifen. These findings indicate that ASPH will serve as a predictor of recurrent triple-negative breast cancer or the HER2-amplified subtype as well as the ER + luminal A or B subtype. Thus, the pathway mediated by ASPH might not be affected by tamoxifen.

Tumor cells employ diverse oncogenically and environmentally driven metabolic pathways to satisfy the bioenergetics and biosynthetic requirements of rapid and sustained growth of tumor cells [25]. Glutamine supports the survival of cancers through its catalytic conversion to glutamate by glutaminase (GLS) in mitochondria $[26,27]$. Glutamate and its metabolites further contribute to rate-limiting cellular metabolic pathways such as the tricarboxylic acid (TCA) cycle, redox, and amino acid synthesis. The survival of triple-negative primary breast cancers and the cognate breast cancer-derived cell lines in vitro is associated with differentially elevated levels of GLSmRNA that support high glutamine utilization and increased dependence on exogenous glutamine [28]. Here we show that higher differential expression of GLS correlated with tumor recurrence in ER + patients with breast cancer, including those treated with tamoxifen. These findings indicate that GLS will serve as a biomarker of recurrence of triple-negative 
breast cancer as well as the ER + luminal A or B subtype. GLS may therefore represent a target of therapy designed to prevent cancer relapse, particularly after lymph nodes metastasis.

The predictive value of our gene expression profile with respect to breast cancer recurrence could be confirmed in larger number of lymph-bide-positive patients or verified in protein level. It might be a new direction that a large number of the same type of receptors as targets, rather than a single target, to develop drugs.

\section{Conclusion}

Sixteen genes encoding proteins with diverse functions and 73 genes encoding olfactory receptors were identified for the first time as potential biomarkers of breast cancer recurrence of patients with the ER + phenotype accompanied by lymph nodes metastasis before surgery. These genes and their products may therefore serve as targets of therapy designed to prevent cancer relapse, particularly after lymph nodes metastasis.

\section{Abbreviation}

formalin-fixed paraffin embedded (FFPE); estrogen receptor (ER); The Kyoto Encyclopedia of Genes and Genomes (KEGG); glutaminase (GLS); Aspartate beta-hydroxylase (ASPH);

\section{Declarations}

\section{Ethical Approval and Consent to participate}

The study was performed in accordance with the guideline laid down in the declaration of Helsinki. All patients gave their informed consent to participate in this study. The Medical Ethics Committee, Shanghai Institute of Planned Parenthood Research, approved the protocols for RNA extraction, microarray hybridization, and data analysis (approval number PJ2019-18).

\section{Consent for publication}

We confirm that this manuscript has not been published elsewhere and is not under consideration in whole or in part by another journal. All authors have approved the manuscript and agree with submission to Breast Cancer Research.

\section{Availability of supporting data}

The microarray data were deposited in the Gene Expression Omnibus (GEO) database (accession code GSE183231).

\section{Competing interests}


The authors declare no competing interests.

\section{Funding}

This work was sponsored by the National Natural Science Foundation of China (81803228).

\section{Authors' contributions}

ZSJ designed the study and helped prepare the manuscript. LY and SSM performed the data analysis. FZ and TX performed the experiments. EL wrote the manuscript.

\section{Acknowledgements}

We thank Dr. Ming Xu of the Central Hospital of Huangpu District, Shanghai for his assistance in the FFPE sample preparation.

\section{References}

1. Wapnir IL, Khan A: Current Strategies for the Management of Locoregional Breast Cancer Recurrence. Oncology (Williston Park) 2019, 33(1):19-25.

2. Wapnir IL, Dignam JJ, Fisher B, Mamounas EP, Anderson SJ, Julian TB, Land SR, Margolese RG, Swain SM, Costantino JP et al: Long-term outcomes of invasive ipsilateral breast tumor recurrences after lumpectomy in NSABP B-17 and B-24 randomized clinical trials for DCIS. J Nat/ Cancer Inst 2011, 103(6):478-488.

3. Santa-Maria CA, Nanda R: Immune Checkpoint Inhibitor Therapy in Breast Cancer. J Nat/ Compr Canc Netw 2018, 16(10):1259-1268.

4. Fisher B, Jeong JH, Anderson S, Bryant J, Fisher ER, Wolmark N: Twenty-five-year follow-up of a randomized trial comparing radical mastectomy, total mastectomy, and total mastectomy followed by irradiation. N Engl J Med 2002, 347(8):567-575.

5. Voduc KD, Cheang MC, Tyldesley S, Gelmon K, Nielsen TO, Kennecke H: Breast cancer subtypes and the risk of local and regional relapse. J Clin Oncol 2010, 28(10):1684-1691.

6. Pan H, Gray R, Braybrooke J, Davies C, Taylor C, McGale P, Peto R, Pritchard KI, Bergh J, Dowsett M et al: 20-Year Risks of Breast-Cancer Recurrence after Stopping Endocrine Therapy at 5 Years. N Engl J Med 2017, 377(19):1836-1846.

7. Wapnir IL, Price KN, Anderson SJ, Robidoux A, Martin M, Nortier JWR, Paterson AHG, Rimawi MF, Lang I, Baena-Canada JM et al: Efficacy of Chemotherapy for ER-Negative and ER-Positive Isolated Locoregional Recurrence of Breast Cancer: Final Analysis of the CALOR Trial. J Clin Onco/2018, 36(11):1073-1079.

8. Paik S, Shak S, Tang G, Kim C, Baker J, Cronin M, Baehner FL, Walker MG, Watson D, Park T et al: A multigene assay to predict recurrence of tamoxifen-treated, node-negative breast cancer. $N \mathrm{Eng} / \mathrm{J}$ Med 2004, 351(27):2817-2826. 
9. King TA, Lyman JP, Gonen M, Voci A, De Brot M, Boafo C, Sing AP, Hwang ES, Alvarado MD, Liu MC et al: Prognostic Impact of 21-Gene Recurrence Score in Patients With Stage IV Breast Cancer: TBCRC 013. J Clin Oncol 2016, 34(20):2359-2365.

10. Poorvu PD, Gelber SI, Rosenberg SM, Ruddy KJ, Tamimi RM, Collins LC, Peppercorn J, Schapira L, Borges VF, Come SE et al: Prognostic Impact of the 21-Gene Recurrence Score Assay Among Young Women With Node-Negative and Node-Positive ER-Positive/HER2-Negative Breast Cancer. J Clin Oncol 2020, 38(7):725-733.

11. Wang Y, Klijn JG, Zhang Y, Sieuwerts AM, Look MP, Yang F, Talantov D, Timmermans M, Meijer-van Gelder ME, Yu J et al: Gene-expression profiles to predict distant metastasis of lymph-node-negative primary breast cancer. Lancet 2005, 365(9460):671-679.

12. Wirapati P, Sotiriou C, Kunkel S, Farmer P, Pradervand S, Haibe-Kains B, Desmedt C, Ignatiadis M, Sengstag T, Schutz F et al: Meta-analysis of gene expression profiles in breast cancer: toward a unified understanding of breast cancer subtyping and prognosis signatures. Breast Cancer Res 2008, 10(4):R65.

13. Desmedt C, Haibe-Kains B, Wirapati P, Buyse M, Larsimont D, Bontempi G, Delorenzi M, Piccart M, Sotiriou C: Biological processes associated with breast cancer clinical outcome depend on the molecular subtypes. Clin Cancer Res 2008, 14(16):5158-5165.

14. Teschendorff AE, Miremadi A, Pinder SE, Ellis IO, Caldas C: An immune response gene expression module identifies a good prognosis subtype in estrogen receptor negative breast cancer. Genome Biol 2007, 8(8):R157.

15. Liang Q, Ma D, Gao RF, Yu KD: Effect of Ki-67 Expression Levels and Histological Grade on Breast Cancer Early Relapse in Patients with Different Immunohistochemical-based Subtypes. Sci Rep 2020, 10(1):7648.

16. Subramanian A, Tamayo P, Mootha VK, Mukherjee S, Ebert BL, Gillette MA, Paulovich A, Pomeroy SL, Golub TR, Lander ES et al: Gene set enrichment analysis: a knowledge-based approach for interpreting genome-wide expression profiles. Proc Natl Acad Sci U S A 2005, 102(43):15545-15550.

17. Pisansky TM, Ingle JN, Schaid DJ, Hass AC, Krook JE, Donohue JH, Witzig TE, Wold LE: Patterns of tumor relapse following mastectomy and adjuvant systemic therapy in patients with axillary lymph node-positive breast cancer. Impact of clinical, histopathologic, and flow cytometric factors. Cancer 1993, 72(4):1247-1260.

18. Weber L, Massberg D, Becker C, Altmuller J, Ubrig B, Bonatz G, Wolk G, Philippou S, Tannapfel A, Hatt $\mathrm{H}$ et al: Olfactory Receptors as Biomarkers in Human Breast Carcinoma Tissues. Front Oncol 2018, 8:33.

19. Xu LL, Stackhouse BG, Florence K, Zhang W, Shanmugam N, Sesterhenn IA, Zou Z, Srikantan V, Augustus M, Roschke $V$ et al: PSGR, a novel prostate-specific gene with homology to a $G$ proteincoupled receptor, is overexpressed in prostate cancer. Cancer Res 2000, 60(23):6568-6572.

20. Neuhaus EM, Zhang W, Gelis L, Deng Y, Noldus J, Hatt H: Activation of an olfactory receptor inhibits proliferation of prostate cancer cells. J Biol Chem 2009, 284(24):16218-16225. 
21. Cui T, Tsolakis AV, Li SC, Cunningham JL, Lind T, Oberg K, Giandomenico V: Olfactory receptor 51E1 protein as a potential novel tissue biomarker for small intestine neuroendocrine carcinomas. Eur $\mathrm{J}$ Endocrinol 2013, 168(2):253-261.

22. Giandomenico V, Cui T, Grimelius L, Oberg K, Pelosi G, Tsolakis AV: Olfactory receptor 51E1 as a novel target for diagnosis in somatostatin receptor-negative lung carcinoids. $J$ Mol Endocrinol 2013, 51(3):277-286.

23. Flegel C, Manteniotis S, Osthold S, Hatt H, Gisselmann G: Expression profile of ectopic olfactory receptors determined by deep sequencing. PLoS One 2013, 8(2):e55368.

24. Lin Q, Chen X, Meng F, Ogawa K, Li M, Song R, Zhang S, Zhang Z, Kong X, Xu Q et al: ASPH-notch Axis guided Exosomal delivery of Prometastatic Secretome renders breast Cancer multi-organ metastasis. Mol Cancer 2019, 18(1):156.

25. Koppenol WH, Bounds PL, Dang CV: Otto Warburg's contributions to current concepts of cancer metabolism. Nat Rev Cancer 2011, 11(5):325-337.

26. Gao P, Tchernyshyov I, Chang TC, Lee YS, Kita K, Ochi T, Zeller KI, De Marzo AM, Van Eyk JE, Mendell JT et al: c-Myc suppression of miR-23a/b enhances mitochondrial glutaminase expression and glutamine metabolism. Nature 2009, 458(7239):762-765.

27. Hensley CT, Wasti AT, DeBerardinis RJ: Glutamine and cancer: cell biology, physiology, and clinical opportunities. J Clin Invest 2013, 123(9):3678-3684.

28. Timmerman LA, Holton T, Yuneva M, Louie RJ, Padro M, Daemen A, Hu M, Chan DA, Ethier SP, van 't Veer LJ et al: Glutamine sensitivity analysis identifies the XCT antiporter as a common triple-negative breast tumor therapeutic target. Cancer Cel/ 2013, 24(4):450-465.

\section{Figures}




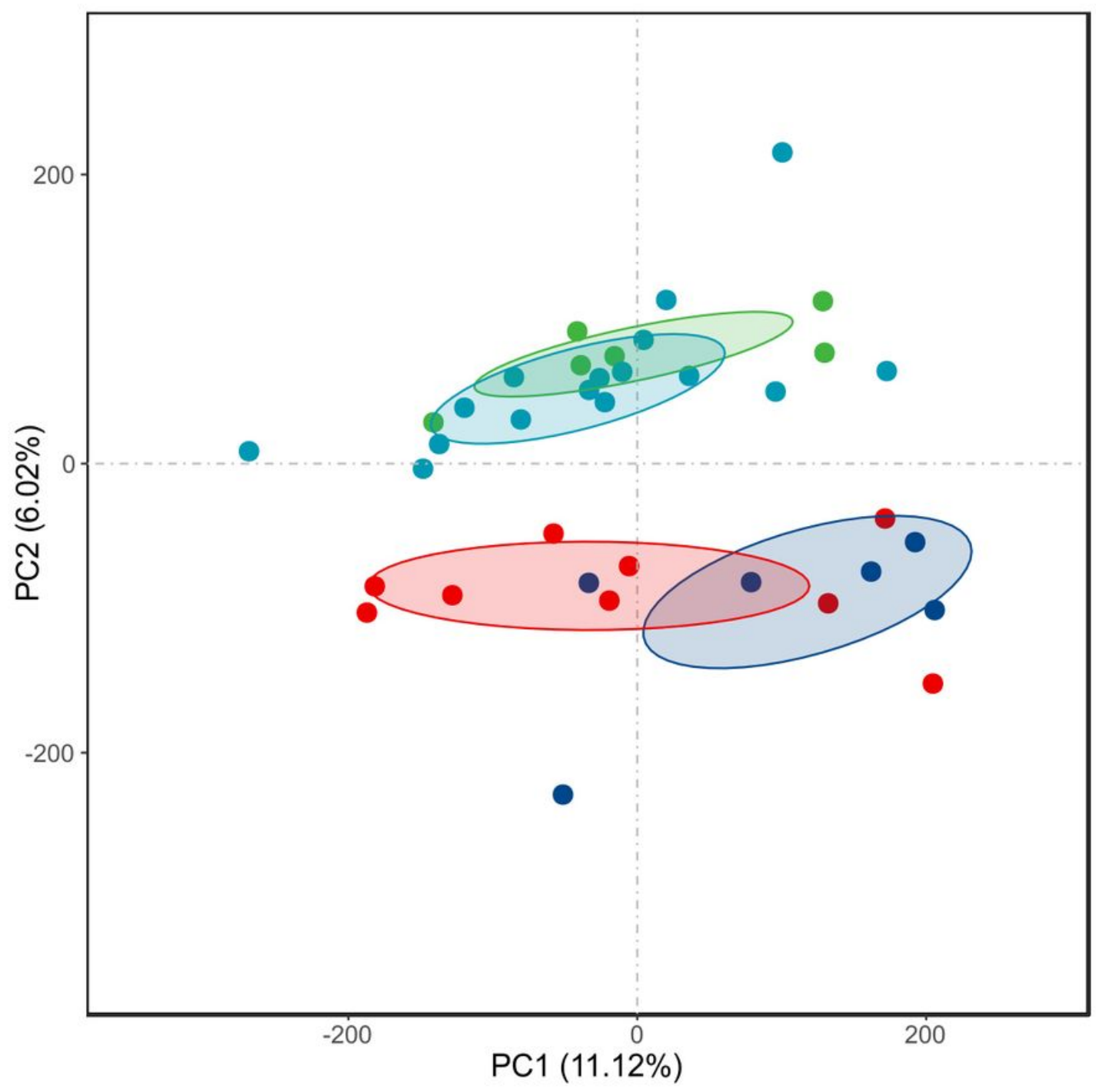

Group

- ER-negative con

- ER-negative recurrence

- ER-positive con

- ER-positive recurrence

\section{Figure 1}

Principal co-ordinates analysis ( $\mathrm{PCOA}$ ) based on gene expression. 

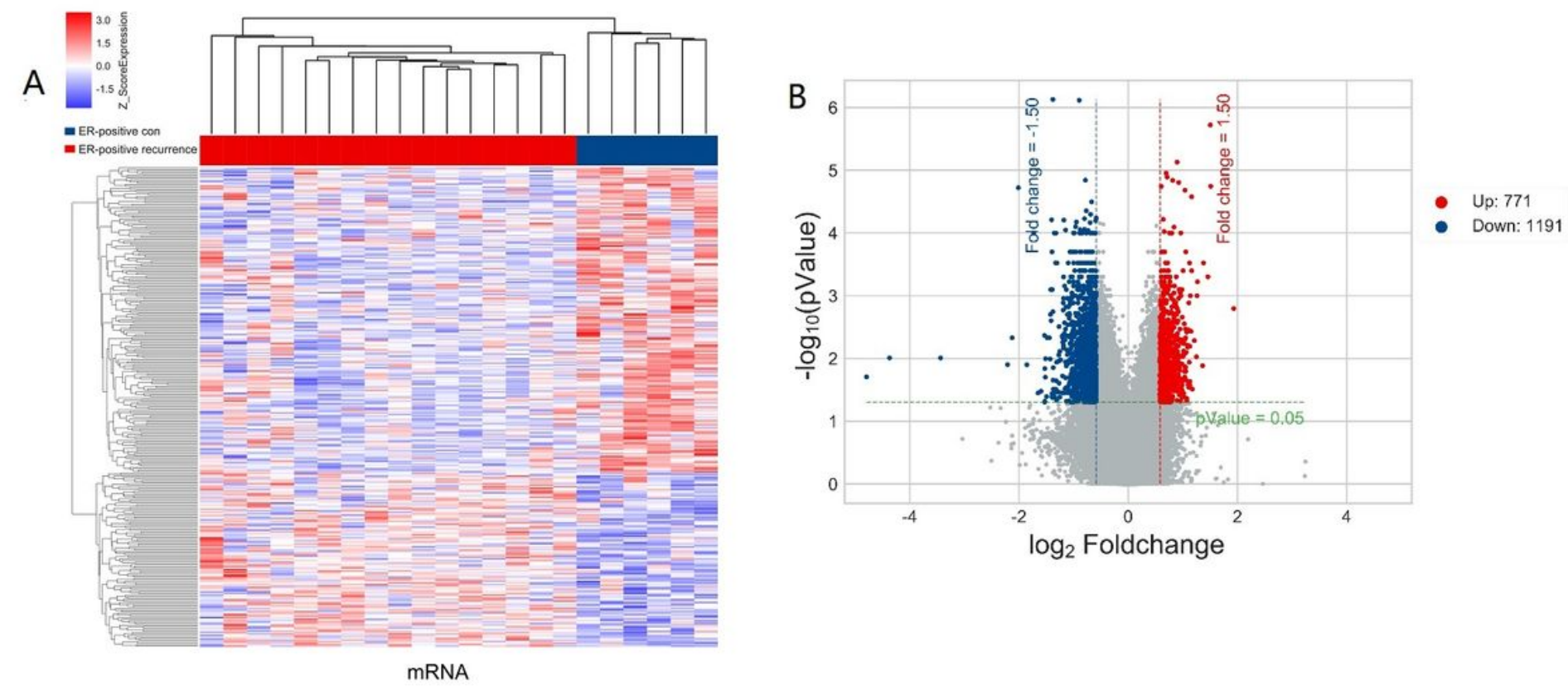

Figure 2

Identification of differentially expressed genes between recurrence and non-recurrence samples of ER+ breast cancer patients. A. The expression heatmap of differentially expressed genes. B. The volcano plot of differentially expressed genes. 
A

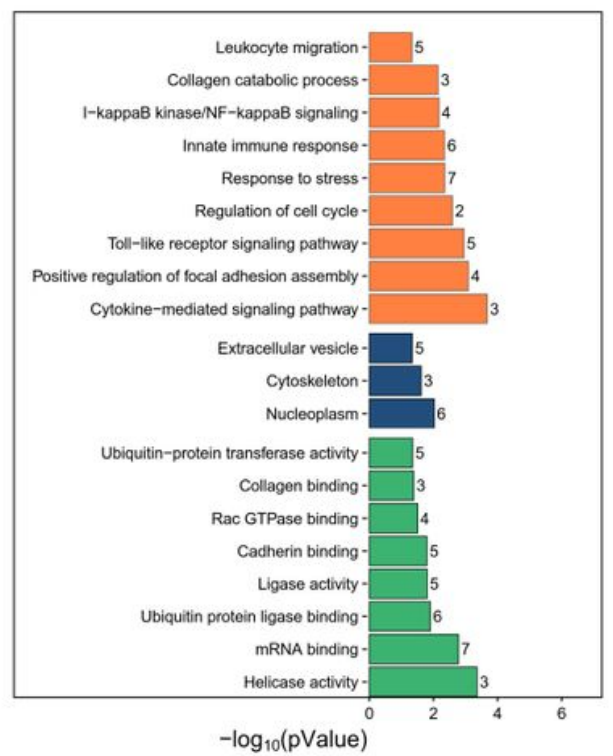

C

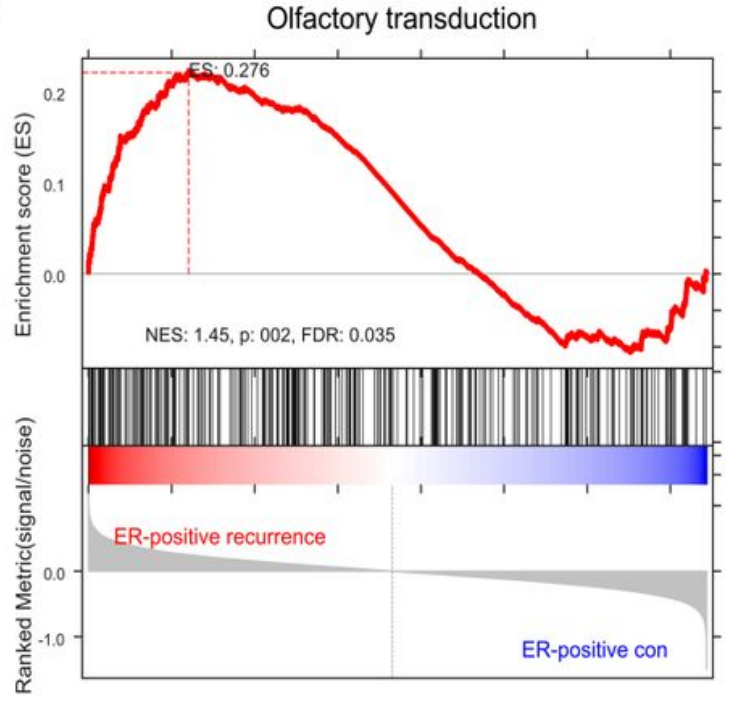

B

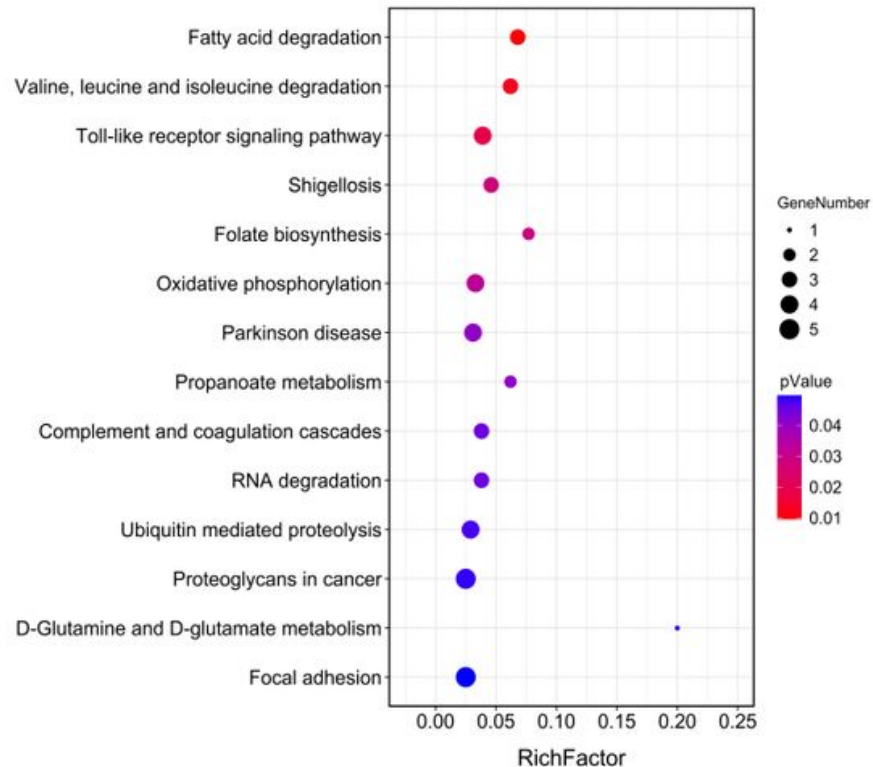

D

DNA binding transcription factor binding

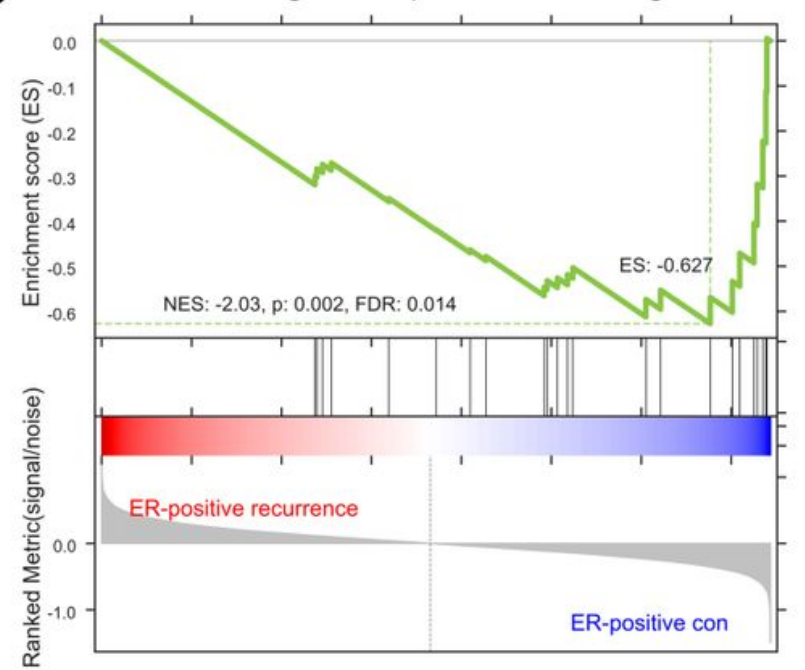

\section{Figure 3}

Functional enrichment analysis of differentially expressed genes between recurrence and non-recurrence samples of ER+ breast cancer patients. A. Top 20 of enriched GO terms. B. Enriched KEGG pathways. C. Enriched KEGG pathway of Olfactory transduction found by GSEA. D. Enriched GO term of DNA binding transcription factor binding found by GSEA. 
A

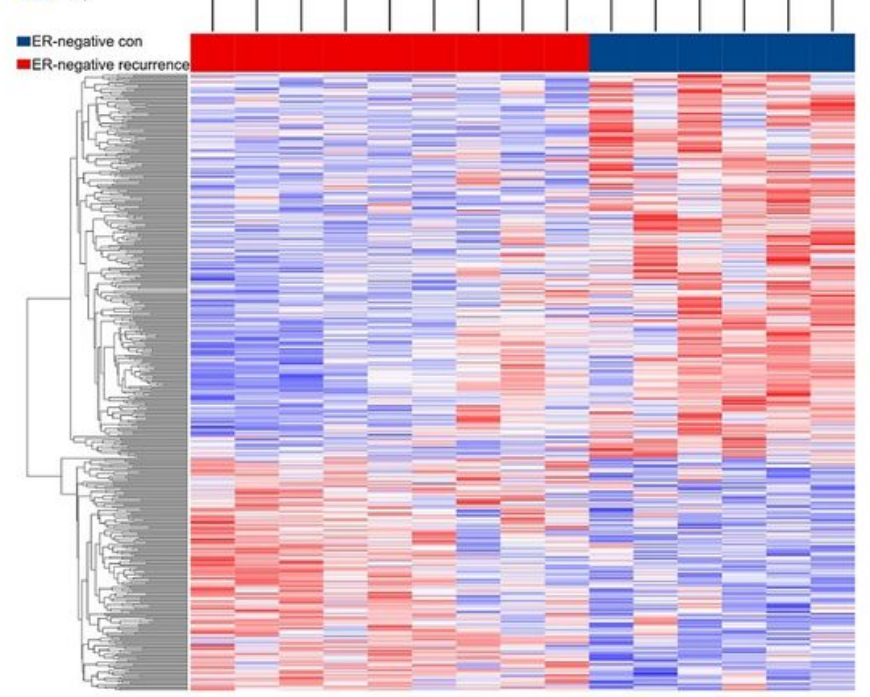

mRNA
B

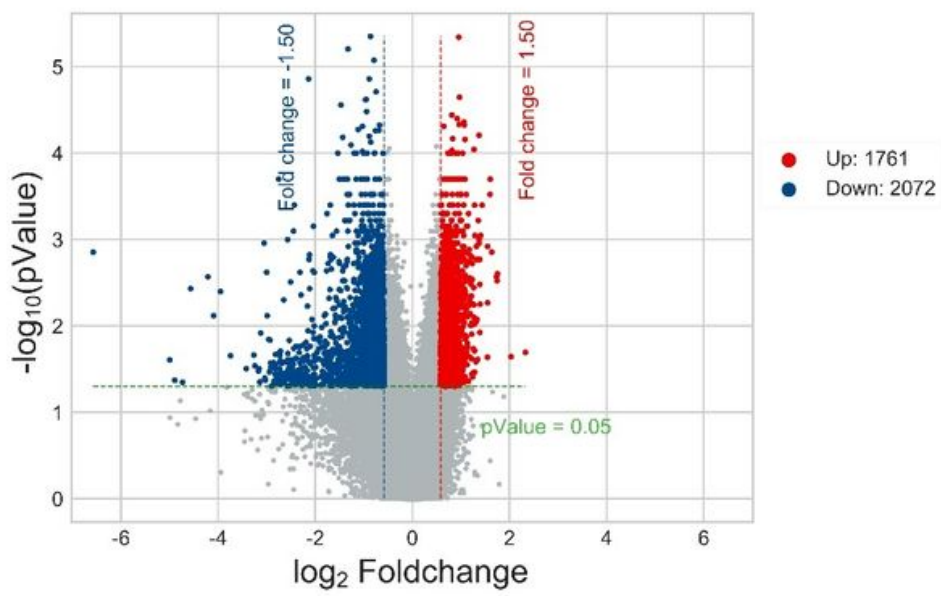

\section{Figure 4}

Identification of differentially expressed genes between recurrence and non-recurrence samples of ERbreast cancer patients. A. The expression heatmap of differentially expressed genes. B. The volcano plot of differentially expressed genes.

A



B



Figure 5 
Functional enrichment analysis of differentially expressed genes between recurrence and non-recurrence samples of ER-breast cancer patients. A. Enriched KEGG pathway of Histidine metabolism found by GSEA. B. Enriched KEGG pathway of Retinol metabolism found by GSEA.

\section{Supplementary Files}

This is a list of supplementary files associated with this preprint. Click to download.

- supplementaryfilesv1.xls 